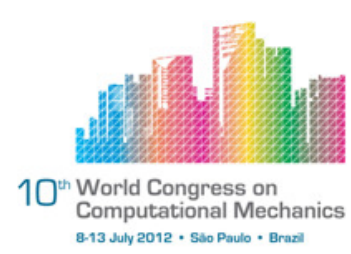

\title{
OPTIMUM DESIGN OF COMPOSITE RISERS USING A GENETIC ALGORITHM
}

\author{
R. F. da Silva, I. B. C. M. da Rocha, E. Parente Jr, A. M. C. de Melo
}

Departamento de Engenharia Estrutural e Construção Civil, Universidade Federal do Ceará. Campus do Pici, Bloco 710, 60455-760, Fortaleza, Ceará, Brasil. (evandro@ufc.br)

\begin{abstract}
Most of recent oil and gas discoveries in Brazil occurred in deepwater fields. Fiber reinforced composite materials present interesting characteristics for offshore applications, such as high specific strength and stiffness, high corrosion resistance, good thermal insulation, high structural damping properties, and fatigue resistance. This work deals with the optimum design of laminated composite risers using a genetic algorithm. The design of laminated composite risers is very difficult since the strength and stiffness of these components depend on the number of layers and the material, thickness, and orientation of each layer. Thus, the use of the conventional trial-and-error strategy is not adequate and it is necessary to apply optimization techniques. In this work the design variables are the number of layers and the thickness and orientation of each layer. A multi-objective formulation is adopted to minimize the weight and maximize the buckling safety factor of the composite riser. The optimization model includes strength and stability constraints and considers multiple load cases. The global analysis of the riser is carried out using the catenary equations and the stress computation in the critical locations is performed using the Classical Lamination Theory (CLT) and the theory of thin-walled tubes. The Tsai-Wu failure criteria is used to compute the safety factor. It is important to note that, due to manufacture constraints, the design variables can only assume discrete values. Therefore, a genetic algorithm is used for optimization since it can easily handle discrete variables. In addition to classical genetic operators, as crossover and mutation, this algorithm also includes operators specially designed to handle laminate structures, such as layer swap and layer deletion. The proposed formulation is applied in the design optimization of composite catenary risers with different water depths and top angles.
\end{abstract}

Keywords: Composite Materials, Risers, Genetic Algorithms.

\section{INTRODUCTION}

Fiber Reinforced Composite (FRC) materials have been widely used in the fabrication of lightweigth structures. Such materials present several advantages over metal equivalents in many applications. Such advantages are high strength and stiffness, low specific weigth, good thermal insulation, chemical resistance and fatigue life. 
In oil drilling, the depletion of existing reserves has increasingly led to the search of deepwater fields. In such environments, the traditional extraction and production risers made of steel tend to become prohibitively expensive, due to increased top tension which overburdens the platform. Composite materials, being lighter, have become an interesting alternative to steel in many different offshore applications [1, 2, 3, 4, 5].

Due to the large number of design variables, including number of layers, thickness and fiber orientation of each layer and material, the traditional trial-and-error procedure can be arduous in the design of fiber reinforced composite risers [6], thus requiring the use of optimization techniques [7].

In the optimization of laminated structures, the design variables can be discrete [8] or continuous $[9,10]$. Usually, no unique solution is obtained, thus leading to the use of multi-objective formulations $[11,12]$. To solve the optimization problem, gradient methods can be used when the variables are continuous [13]. Evolutionary algorithms have also seen extensive use [14, 15, 16], particularly Genetic Algorithms [17, 18]. Such algorithms readily deal with discrete variables and do not tend to get trapped in local minima.

In dealing with constrained problems using genetic algorithms, some method has to be used to enforce the constraints, since the algorithm does not treat them directly. To this end, adaptive penalty methods are interesting alternatives to the commonly used constant penalty strategy. The interaction between such penalty methods and the selection and fitness function strategies are analyzed in this work for the case of composite catenary riser optimization.

In the present work, a genetic algorithm with special laminate operators is used to design composite risers in catenary configuration. The design variables are the thickness and fiber orientation of each layer. A multi-objective function involving the composite crosssectional area and the buckling safety factor is used. The formulation also includes stability and strength constraints and can deal with multiple load cases. The structural analysis is performed by the Classical Lamination Theory and failure is predicted using the Tsai-Wu failure criteria.

\section{STRUCTURAL ANALYSIS}

The mechanical behavior of each fiber reinforced lamina is readily defined as an homogeneous and orthotropic material in its local coordinate system $(1,2,3)$ when analyzing the material in a macroscopic scale. However, as each lamina has its own local system, a global coordinate system is devised $(\mathrm{x}, \mathrm{y}, \mathrm{z})$ to which the properties of all laminae are transformed. Both systems are equally important, since the equilibrium equations are written in the global system but the evaluation of failure criteria is done in the local system for each ply [19]. Both systems are shown in Figure 1.

In the definition of the local coordinate system, 1 is the direction of the fibers, 2 is the in-plane direction normal to the fibers and 3 is the out of plane normal to the lamina. The local and global systems are related by the angle $\theta$ between the $\mathrm{x}$-axis and 1-axis, as shown in Figure 1.

Experimental evidence shows that a fiber reinforced lamina behaves as a physically linear material until very close to failure. Thus, the relation between stresses $\sigma_{1}$ and strains 


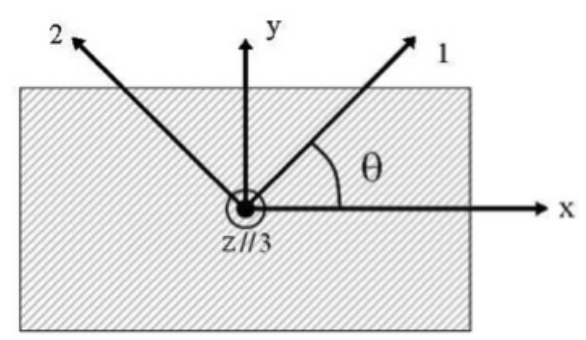

Figure 1. Local and global coordinate systems [20].

$\boldsymbol{\epsilon}_{1}$ in the local system $(1,2,3)$ can be modeled using Hooke's Law in a plain stress condition:

$$
\left[\begin{array}{l}
\sigma_{1} \\
\sigma_{2} \\
\sigma_{6}
\end{array}\right]=\left[\begin{array}{ccc}
C_{11} & C_{12} & 0 \\
C_{12} & C_{22} & 0 \\
0 & 0 & C_{66}
\end{array}\right]\left[\begin{array}{l}
\epsilon_{1} \\
\epsilon_{2} \\
\epsilon_{6}
\end{array}\right] \Rightarrow \boldsymbol{\sigma}_{\mathbf{1}}=\mathbf{C} \boldsymbol{\epsilon}_{\mathbf{1}}
$$

where the coefficients of the constitutive matrix $\mathbf{C}$ are

$$
C_{11}=\frac{E_{1}}{1-\nu_{12} \nu_{21}} \quad C_{12}=\frac{\nu_{12} E_{2}}{1-\nu_{12} \nu_{21}} \quad C_{22}=\frac{E_{2}}{1-\nu_{12} \nu_{21}} \quad C_{66}=G_{12}
$$

To transform the mechanical properties of each lamina from the local to the global coordinate system, one has to establish a relation between the two systems. Using the lamination angle $\theta$ showed in Figure 1, a strain transformation from the global to the local coordinate system can be written as [21]:

$$
\left[\begin{array}{l}
\epsilon_{1} \\
\epsilon_{2} \\
\epsilon_{6}
\end{array}\right]=\left[\begin{array}{ccc}
\cos ^{2} \theta & \sin ^{2} \theta & \sin \theta \cos \theta \\
\sin ^{2} \theta & \cos ^{2} \theta & -\sin \theta \cos \theta \\
-2 \sin \theta \cos \theta & 2 \sin \theta \cos \theta & \cos ^{2} \theta-\sin ^{2} \theta
\end{array}\right]\left[\begin{array}{c}
\epsilon_{x} \\
\epsilon_{y} \\
\epsilon_{x y}
\end{array}\right] \Rightarrow \boldsymbol{\epsilon}_{\mathbf{1}}=\mathbf{T} \boldsymbol{\epsilon}
$$

Using the Virtual Work Principle, the stresses in the global system can be obtained from those in the local system using the following transformation [21]:

$$
\sigma=\mathrm{T}^{\mathrm{T}} \boldsymbol{\sigma}_{1}
$$

From Eqs. (3) and (4), the constitutive relation expressed in Eq. (1) can be transformed to the global system:

$$
\sigma=\bar{C} \epsilon \Rightarrow \bar{C}=\mathrm{T}^{\mathrm{T}} \mathrm{CT}
$$

where the term $\bar{C}$ is the transformed constitutive matrix and the transformation matrix $\boldsymbol{T}$ is the one shown in Eq. (3).

With the constitutive relation in the global coordinate system, the Classical Lamination Theory [22] can be used to obtain strains at planes parallel to the laminate mid-surface:

$$
\boldsymbol{\epsilon}=\boldsymbol{\epsilon}_{\boldsymbol{m}}+z \kappa
$$

where $\epsilon_{m}$ are the membrane strains, $\kappa$ represents the curvatures of the laminate and $\mathrm{z}$ is the distance from the mid-surface. Integrating the global stresses through the laminate thickness $(t)$, the resultant forces are obtained:

$$
\mathbf{N}=\left\{\begin{array}{c}
N_{x} \\
N_{y} \\
N_{x y}
\end{array}\right\}=\int_{-t / 2}^{t / 2}\left\{\begin{array}{c}
\sigma_{x} \\
\sigma_{y} \\
\tau_{x y}
\end{array}\right\} d z \quad \mathbf{M}=\left\{\begin{array}{c}
M_{x} \\
M_{y} \\
M_{x y}
\end{array}\right\}=\int_{-t / 2}^{t / 2}\left\{\begin{array}{c}
\sigma_{x} \\
\sigma_{y} \\
\tau_{x y}
\end{array}\right\} z d z
$$


Using Eqs. (5), (6) and (7), one can obtain the resultant forces in terms of the global strains, summing up the contribution of each lamina:

$$
\left\{\begin{array}{l}
\mathrm{N} \\
\mathrm{M}
\end{array}\right\}=\left[\begin{array}{ll}
\mathrm{A} & \mathrm{B} \\
\mathrm{B} & \mathrm{D}
\end{array}\right]\left\{\begin{array}{c}
\epsilon_{m} \\
\kappa
\end{array}\right\}
$$

where $\mathbf{A}$ is the membrane (or extension) stiffness matrix, $\mathbf{D}$ is the bending stiffness matrix and $\mathbf{B}$ is the bending-extension coupling stiffness matrix. The coefficients of such matrices are given by:

$$
A_{i j}=\sum_{k=1}^{n} \overline{\boldsymbol{C}}_{i j}^{k}\left(z_{k+1}-z_{k}\right) \quad B_{i j}=\sum_{k=1}^{n} \frac{\overline{\boldsymbol{C}}_{i j}^{k}\left(z_{k+1}^{2}-z_{k}^{2}\right)}{2} \quad D_{i j}=\sum_{k=1}^{n} \frac{\overline{\boldsymbol{C}}_{i j}^{k}\left(z_{k+1}^{3}-z_{k}^{3}\right)}{3}
$$

It is important to note that if the laminate is symmetric, as is the case of all numeric examples in this paper, $\mathbf{B}=\mathbf{0}$ and there is no membrane-bending coupling, meaning that no bending strains come with the application of only membrane loads. However, if the laminate is not symmetric, such coupling occurs and bending strains are generated by membrane loads.

\subsection{Global Analysis}

In the present work, the global analysis procedure is undertaken by an analytical catenary solver. The main advantage of such approach is its speed and result reliability. However, to better represent the internal forces to which the composite riser is subjected during service, a nonlinear time-domain dynamic finite element model should be used. Such method would be much slower and since the main objective of this work is to assess the efficiency of genetic algorithms in composite riser optimization, the simplified catenary model was chosen.

Thus, the riser is considered an inextensible cable subjected to a vertical weigth load distributed along its length [23]. Such load also includes the internal fluid weigth and is based on the apparent weigth of the riser as it is immersed in water:

$$
w_{\text {wet }}=w_{\text {dry }}+w_{\text {fluid }}-w_{\text {water }}
$$

where $w_{d r y}$ is the riser dry weigth, $w_{\text {fluid }}$ is the weigth of internal fluid and $w_{\text {water }}$ is the weigth of the displaced water, accounting for the buoyancy force.

Three different floater positions, Mean, Near and Far, are used in the analysis procedure, accounting for the platform movement. The Mean position is defined by the top angle $\alpha$ related to the vertical. The Near and Far positions are defined simply by the platform offset $\Delta$ with respect to the Mean position. Such parameters are shown in Figure 2.

The axial force computed using Eq. 10 is known as the effective tension $\left(N_{e f}\right)$, but it does not account for the effect of hydrostatic pressure [23] and thus has to be properly modified, resulting in the true wall tension $\left(N_{t w}\right)$ :

$$
N_{t w}=N_{e f}+p_{i} A_{i}-p_{e} A_{e}
$$

where $p_{i}$ is the internal pressure, $A_{i}$ is the internal area, $p_{e}$ is the external pressure and $A_{e}$ is the external area. 


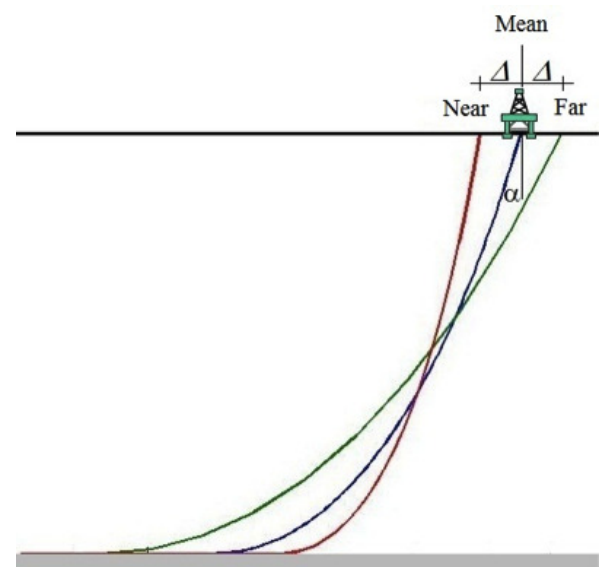

Figure 2. Catenary riser positions.

As the catenary model is static and handles only axial forces $(\mathbf{M}=\mathbf{0})$, an Amplification Factor $\beta$, applied in the effective tension $N_{e f}$ is used to take into account bending, torsion and dynamic effects caused by wave and current loads. The normal forces are then given by:

$$
\begin{gathered}
N_{x}=\frac{\gamma_{f} N_{t w}}{2 \pi R} \\
N_{y}=\gamma_{f} p_{i} R_{i}-\gamma_{f} p_{e} R_{e} \\
N_{x y}=0
\end{gathered}
$$

where $R, R_{i}$ and $R_{e}$ are the mean, inner and outer radius of the tube, respectively. It should be noted that $N_{e f}, p_{e}$ and $p_{i}$ are multiplied by the load factor $\gamma_{f}$ to obtain the design forces in a semi-probabilistic approach [24].

According to Eq. (8), for symmetric laminates, $\mathbf{B}=\mathbf{0}$ and there is no extensionbending coupling. Also, since there are no bending moments, the bending strain $\kappa$ is also zero. The strains in the laminate are then computed from:

$$
\left\{\begin{array}{c}
\mathrm{N} \\
0
\end{array}\right\}=\left[\begin{array}{cc}
\mathrm{A} & 0 \\
0 & \mathrm{D}
\end{array}\right]\left\{\begin{array}{c}
\epsilon_{m} \\
\kappa
\end{array}\right\} \Rightarrow\left\{\begin{array}{l}
\epsilon_{m}=\mathrm{A}^{-1} \mathrm{~N} \\
\kappa=0
\end{array} \Rightarrow \epsilon=\epsilon_{m}\right.
$$

With the global strains, the transformation to the local system is done using Eq. (3) and the corresponding local stresses are calculated using Eq. (1). Such stresses are then used to evaluate structural safety using the failure criteria presented below.

\subsection{Stability Analysis}

Deepwater risers are subjected to high hydrostatic pressures during service life. Such external pressures cause high compressive stresses in the tube wall, which can lead to a local buckling collapse well below the material strength. As this is a major concern in deepwater pipelines, a local buckling constraint has to be considered in the optimization process.

The critical pressure for hoop buckling of long orthotropic cylindrical shells $[25,26]$ can be computed from:

$$
p_{c r}=\frac{3}{R^{3}}\left(D_{22}-\frac{B_{22}^{2}}{A_{22}}\right)
$$


where $A_{22}, B_{22}$ and $D_{22}$ are computed using the Classical Lamination Theory through Eq. (9). It is well-known that geometric imperfections reduce the final load bearing capacity of cylindrical shells. Therefore, the collapse pressure which is used in the design optimization process is given by:

$$
p_{c o l}=k_{p} p_{c r}
$$

where $k_{p}$ is a knock-down factor accounting for the effect of geometric imperfections settled as 0.75 [25].

\section{GENETIC ALGORITHM}

In nature, individuals must have useful genetic features in order to survive. Such individuals are the fittest and thus have more chances of passing its characteristics to future generations, while those less fit succumb along the evolution process.

Such natural process is mimicked by the so-called Genetic Algorithms (GA) in order to solve optimization problems. As the concept of population is used, such algorithms work with a set of potential solutions.

GAs present many advantages that have led to a rise in their use, particularly with the rise in the processing power of common computers. As they work with a set of potential solutions, GAs do not easily get trapped in local minima. Furthermore, since no gradient data is needed, GAs can deal with discontinuous design spaces and problems with discrete variables and non-differentiable functions.

One of the main difficulties of working with GAs is the consideration of constrained problems, since they cannot be explicitly treated. Several options exist in order to permit the solution of constrained optimization problems, many of which are presented in [27]. Some of them are presented here and used in the optimization of composite risers.

The genetic algorithm developed in this work uses well-established genetic operators such as crossover and mutation, but also features some operators especially designed to treat the problem of laminated composites.

The general flow of the optimization process using a genetic algorithm is as follows. Initially, a random starting population is generated. Then, the fitness function is calculated and some sort of selection process is used to generate the mating pool, with the same number of individuals as the initial population. From the mating pool, some individuals are selected for breeding, generating an offspring population. Then, mutation and other operators are applied to such offspring and they form the new population, with the remaining members being filled by elitism from the initial population. Afterwards, the process is repeated until a certain number of generations is reached or the objective function of the best individual remains unchanged during a user-defined number of generations (stallgen).

\subsection{Encoding}

Each trial laminate is an individual represented by two chromosomes in matrix form. The thickness chromosome stores information about thickness and the orientation chromosome stores data about the fiber orientation angles. In each of the chromosomes, a pair of genes represents a layer. 
Each laminate has a genotypical and phenotypical representation. On the genotype, chromosomes have integer values in each gene, corresponding to list positions which store discrete values of the design variables. Thus, the algorithm used in this work features integer coding. The phenotype representation is used to store the design variables of a design.

Figure 3 shows an example of codification from genotype to phenotype representation. Layers which were removed by the ply deletion operator are given zero values in both chromosomes for the respective layer.

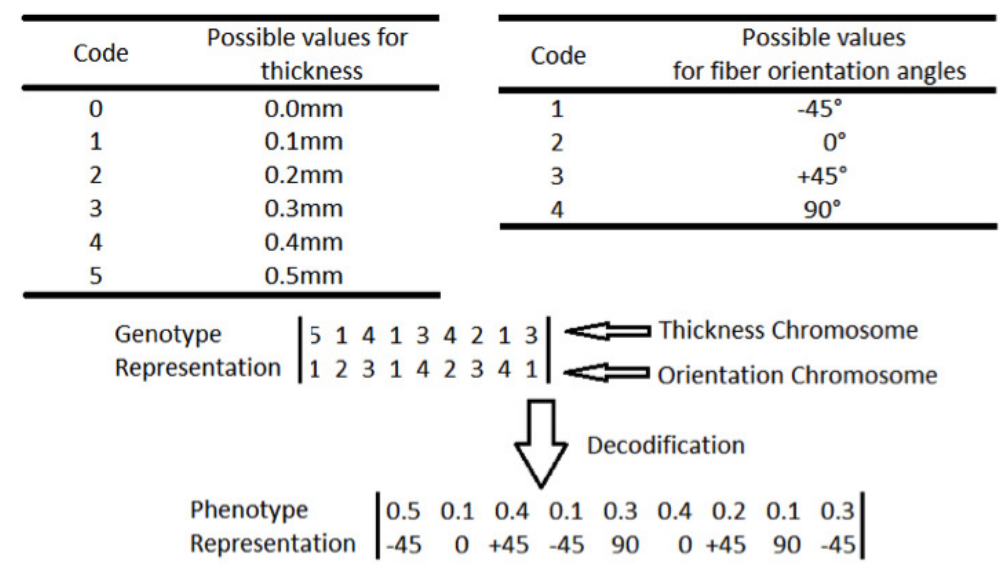

Figure 3. Genotype to phenotype decodification example.

\subsection{Penalty Methods}

As mentioned earlier, Genetic Algorithms do not handle constraints explicitly. Thus, to solve constrained problems, some method has to be used in order to maintain the population, or most of it, in the feasible space. To this end, several methods have been devised, some of which were presented in [27].

In direct (or interior) methods, only feasible points are allowed in the design process. The simplest way to force satisfaction of constraints is the so-called Death Penalty method [27], in which infeasible points are simply discarded and new individuals are generated until all members of the population are viable. This method can be computationally inefficient and will most likely eliminate infeasible individuals with good genetic characteristics, which could accelerate the algorithm convergence.

Another alternative to directly apply the constraints is to use a coding scheme that always generates feasible phenotypes, no matter which genotype is given. Such strategy can be efficient, but the coding scheme would be specific for each problem, which can be cumbersome. Also, only explicit constraints can be treated in such a way. Repair techniques, which bring an infeasible point back to the feasible region, and special crossover operators can be devised to maintain feasibility. However, such methods also heavily depend on the problem formulation.

Indirect (or exterior) methods are the most used. They permit the presence of infeasible individuals in the pupulation, but their objective function is penalized making them less fit and thus less capable of transmitting genes to next generations. It is important to note that many of the well-known indirect penalty methods require the analyst to provide one or 
more penalty parameters, which can greatly vary between problems or even between multiple executions of the same problem. Thus, methods that do not require any parameters become advantageous and will be focused in the present section.

\subsubsection{Constant Penalty}

This is the simplest indirect method to treat constraints. It consists in defining a constant penalty factor $k$, usually a very high number provided by the user, and use it to penalize infeasible individuals based on their constraint violations. Using such method, the penalized objective function for a minimization problem in which all constraints are written in the form $g_{l}(\mathbf{x}) \leq 0$ is given by:

$$
f_{p}=f(\mathbf{x})+\sum_{l=1}^{m} k \cdot \max \left(g_{l}(\mathbf{x}), 0\right)
$$

In the above expression, all constraints are treated using the same $k$. Thus, it is always recommended to use non-dimensionalized constraints, as they can be of different orders of magnitude. As noted earlier, the determination of an acceptable $k$ value can result in a trialand-error process, as it heavily depends on the problem formulation. Usually, a very high number is used, which can lead to numerical problems depending on the order of magnitude of the design variables, objective function and constraints. Therefore, even though a constant penalty is easily implemented, adaptive methods in which no user-defined penalty factors are needed can help overcome such disadvantages.

\subsubsection{Deb's Method}

The following method was proposed by Deb [28] and was coupled with a tournament selection method. Here, infeasible individuals are first penalized until they have the same objective function as the worst individual in the population $\left(f_{\max }\right)$. Then, the sum of constraint violations is added:

$$
f_{p}= \begin{cases}f(\mathbf{x}) & \text { if } \mathbf{x} \text { is feasible } \\ f_{\text {max }}+\sum_{l=1}^{m} g_{l}(\mathbf{x}) & \text { if } \mathbf{x} \text { is infeasible }\end{cases}
$$

The main advantage of Deb's Method resides in the fact that no penalty parameter is required. Also, the method is of easy computational implementation. It is important to note, however, that the efficiency of the method also depends on the selection method and the type of genotype representation [27].

It seems to the authors that, if tournament selection is used, the only way for an infeasible individual to go into the mating pool is to be chosen alongside another infeasible individual in tournament. However, if a fitness proportional selection is used, they may have greater chances of getting into the mating pool. Therefore, the combination of Deb's Method and tournament selection seems rather strict with infeasible individuals. 


\subsubsection{Adaptive Penalty}

The adaptive penalty method used in the present work was proposed by Barbosa and Lemonge [27]. It involves separate penalty factors for each constraint. Also, such factors $\left(k_{j}\right)$ have different values in each generation.

First, the objective function of all infeasible individuals are taken at least to the mean value for the population. This process is similar to the one in Deb's Method, but the individuals are not taken straight to the maximum value for the population (Section 3.2.2). Thus, defining $f_{m}$ as the mean objective function of the current population, $\bar{f}$ is defined as:

$$
\bar{f}(\mathbf{x})= \begin{cases}f(\mathbf{x}) & \text { if } f(\mathbf{x})>f_{m}(\mathbf{x}) \\ f_{m}(\mathbf{x}) & \text { if } f(\mathbf{x})<f_{m}(\mathbf{x})\end{cases}
$$

To define each constraint coefficient $k_{j}$, the mean violation $g_{m}$ of each constraint is defined, as well as the quadratic sum of all mean violations. Each $k_{j}$ is then defined as:

$$
k_{j}=\left|f_{m}\right| \frac{g_{m(j)}}{\sum_{l=1}^{m} g_{m(l)}^{2}}
$$

It is important to note that for those constraints with the highest mean violation, the corresponding coefficients $k_{j}$ are bigger. In other words, the constraints for which the population is having the most difficulty to satisfy are the most penalized [27]. With the definition of $\bar{f}$ given in Eq. (20) and $k_{j}$ given in Eq. (21), the penalized objective function can be defined:

$$
f_{p}(\mathbf{x})= \begin{cases}f(\mathbf{x}) & \text { if } \mathbf{x} \text { is feasible } \\ \bar{f}(\mathbf{x})+\sum_{j=1}^{m} k_{j} \cdot \max \left(g_{j}(\mathbf{x}), 0\right) & \text { if } \mathbf{x} \text { is infeasible }\end{cases}
$$

This method does not require any input from the user, and the penalty is adjusted with the generations. Comparing the method with Deb's one (Section 3.2.2), the latter seems harsher with infeasible individuals. It is expected that, for most problems, the maximum penalty factor obtained adaptively will be smaller than the large $k$ used in the constant penalty method.

\subsection{Fitness Function and Selection}

For the purpose of selection to the mating pool and subsequent selection for crossover, individuals need be assigned a positive numerical value that represents its fitness in the current population. Such value dictates how an individual is better or worse than others.

In some maximization problems, the penalized objective function can assume only positive values and can directly be used as the fitness function. However, in several problems, a objective function must be minimized instead of maximized and this function is not restricted to assume only positive values. As a result, the penalizedobjective function should be mapped to a fitness function in order to ensure that the fitness will have a positive value for all individuals. Mapping must also guarantee that for a individual, the bigger its objective 
function value, the smaller its fitnesses will be [29]. Some methods of mapping the objective into a fitness function can be found in [30]. In this work, the linear scaling mapping, given by:

$$
\text { Fit } t_{i}=f_{s c}-f_{p i}, \quad \text { where } f_{s c}=\max \left(\left|f_{p(\min )}\right|,\left|f_{p(\max )}\right|\right)
$$

is used. Fit $t_{i}$ is the fitness of the $i-t h$ individual. $f_{p(\min )}$ and $f_{p(\max )}$ are the minimum and maximum penalized objective function values in the current population. $f_{p i}$ is the penalized objective function value of the $i-t h$ individual.

Using the fitness scores, individuals are choosen to breed, originating the offspring. From the several commonly selection methods at dispostal in literature ([31]), the Fitness Proportinal Selection and Ranking Selection are employed here. The first method assigns for each individual a probability of being selected directly proportional to its fitness. The second method first ranks all individuals based on their fitness and assigns a probability of being selected directly proportional to their positions in the rank. Comparing to Ranking, the Fitness Proportinal Selection tends be more sensible to a change in the penalty method (Section 3.2), since the absolute differences between individuals are taken into account.

It is important to note that once an individual is chosen, it is not eliminated from the selection process, thus giving the chance for the same individual to be selected multiple times.

\subsection{Crossover}

Crossover is a key genetic operator and is the main responsible for the convergence of a genetic algorithm. Such operator is applied on two individuals, called parents, and originates two new individuals called sons, which contain the combined traits of the parents. Parents are taken from the mating pool, which is filled with individuals of the original population using the selection procedure. The number of parents selected for crossover is dictated by the crossover rate.

In this work, the crossover consists in a linear combination of the parents traits in order to form the sons. The process is done layer by layer, with a random $r$ number between 0 and 1 been chosen. Such random number is then used to calculate a weighted mean of the parents genes, rounded to the closest integer. Figure 4 shows an example of the process in which two parents, each containing three layers, generate two sons.

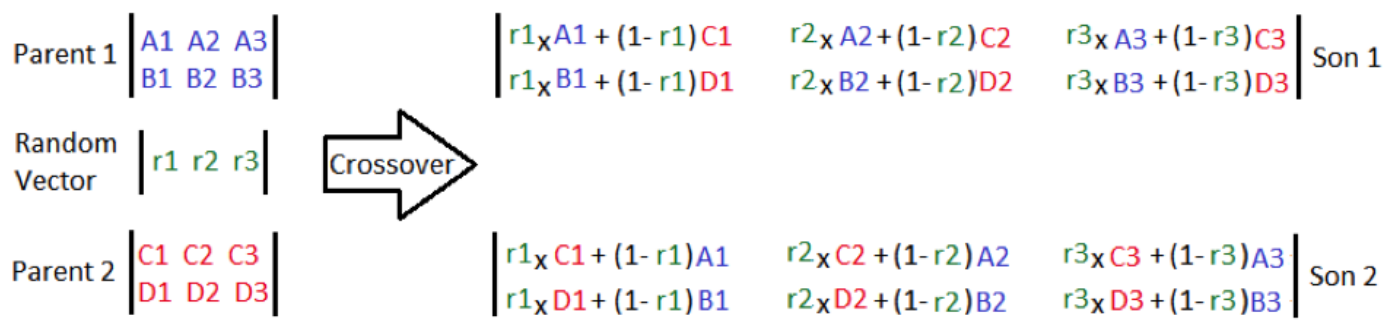

Figure 4. Crossover operator applied on two 3-layer laminates.

\subsection{Mutation}

Even though genetic algorithms have lesser chances to be trapped in local minima, sometimes a premature convergence can occur. To prevent it, genetic variability has to be 
maintained. The mutation operator is one of the strategies used to ensure variability within the population, and it is applied in the sons generated by the crossover process. It has a low probability of occurring, called mutation rate. For each gene (layer) of the chromosome, a random number is generated. If such number is lower than the mutation rate, a random integer replaces the original gene value. Figure 5 shows mutation applied on two genes.

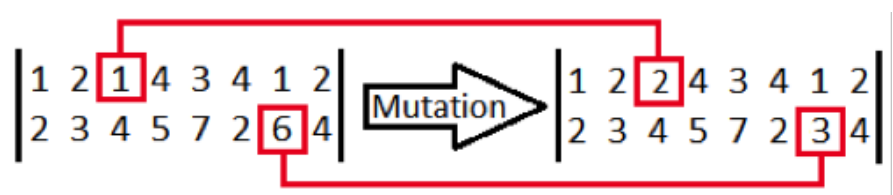

Figure 5. Mutation operator applied on two genes of a chromosome.

\subsection{Laminate Operators}

The gene-swap, or layer-swap operator changes the mechanical properties of the laminate by exchanging the position of two layers. As with the mutation operator, the layer-swap has a low probability of occurring. The choice of plies to be exchanged are also randomized. It is important to note that, according to Eq. (9), the layer-swap operator changes the D matrix without changing the A matrix. This can be useful in improving the external pressure buckling resistance of the riser. Figure 6 shows an example of gene-swap.

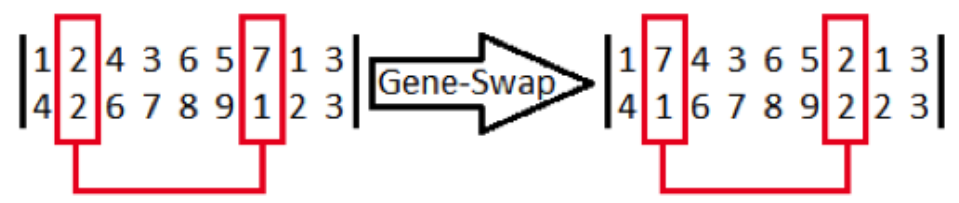

Figure 6. Example of the layer-swap operator.

The ply deletion operator is similar to a mutation. For each layer, a random number between 0 and 1 is generated. If it is smaller than the deletion rate, the thickness of such layer is set as 0 , thus effectively eliminating the layer. Similarly, the ply addition is applied in all genes who were previously deleted or at the end of the laminate. If the addition rate is satisfied, a random thickness and orientation angle are inserted, effectively adding a layer. Figure 7 shows examples of ply deletion and addition.

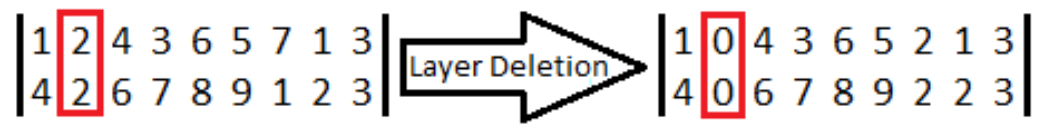

$$
\begin{aligned}
& \left|\begin{array}{llllllll}
1 & 2 & 4 & 3 & 6 & 5 & 7 & 1 \\
4 & 2 & 6 & 7 & 8 & 9 & 1 & 2
\end{array}\right| 0 \mid \text { Layer Addition }\left|\begin{array}{llllllll}
1 & 2 & 4 & 3 & 6 & 5 & 2 & 1 \\
4 & 2 & 6 & 7 & 8 & 9 & 2 & 2
\end{array}\right|
\end{aligned}
$$

Figure 7. Layer addition and deletion. 


\section{RISER OPTIMIZATION MODEL}

The input data for the riser problem includes material properties, loads, maximum number of layers $(n)$, inner radius $\left(R_{i}\right)$, minimum and maximum ply thickness, steel liner thickness, insulation layer thickness, minimum and maximum lamination angle $(\theta)$, minimum safety factors for each material, amplification factor $(\beta)$, force coefficient $\left(\gamma_{f}\right)$, top angle, floater offset and water depth.

Input data specific to the genetic algorithm includes number of generations, population size, number of generations for stallgen, penalty method, and fitness function method. Also, the rates for crossover, mutation, layer-swap, ply addition and ply deletion are given.

The design variables are the thickness $(t)$ and orientation angle $(\theta)$ of each ply, with only symmetric lay-ups being allowed. Therefore, only half of the laminate is stored as design variables:

$$
\mathbf{x}=\left\{t_{1}, t_{2}, \ldots, t_{n / 2} \quad \theta_{1}, \theta_{2}, \ldots, \theta_{n / 2}\right\}
$$

Due to manufacturing constraints, most works dealing with optimization of laminated structures make use of discrete design variables [32, 33]. Usually, the manufacturing process does not permit small angle variations. Also, the ply thickness is commonly a multiple of some predefined value [34]. However, continuous variables have also been used [35], yielding results that can lead to lower boundaries to a more constrained discrete problem [36]. In this work, only discrete variables are used:

$$
\begin{aligned}
& \theta_{p}=\left\{\theta_{\text {min }}, \theta_{\text {min }}+\Delta \theta, \theta_{\text {min }}+2 \Delta \theta, \ldots, \theta_{\text {max }}\right\} \\
& t_{p}=\left\{t_{\text {min }}, t_{\text {min }}+\Delta t, t_{\text {min }}+2 \Delta t, \ldots, t_{\text {max }}\right\}
\end{aligned}
$$

where $\Delta \theta$ and $\Delta t$ are the angle and thickness increments, respectively.

The composite riser cost is considered directly proportional to the composite crosssection area, which is constant along the riser length. Therefore, the objective function could be initially defined as the composite cross-sectional area. However, for certain water depths, many different solutions are obtained with the same area, but with different buckling loads. In this case, a multi-objective function also involving the buckling safety factor will be used:

$$
f(\mathbf{x})=w_{1} \frac{A_{\min }-A}{A_{\min }-A_{\max }}+\left(1-w_{1}\right) \frac{S F_{\text {buck }(\max )}-S F_{\text {buck }}}{S F_{\text {buck }(\max )}-S F_{\text {buck }(\min )}}
$$

where both the area and the buckling safety factor are normalized by their minimum and maximum values. Such values for the buckling safety factor are determined considering a minimum thickness laminate with only $0^{\circ}$ plies for the minimum SF and a maximum thickness laminate with only $90^{\circ}$ plies for the maximum SF.

The authors in [37] also used buckling loads in the objective function. In [8], many different parameters were used in the objective functions, such as the weigth, deflection, cost and stiffness of the laminate. If the problem involves plates, the total thickness can also be used. Therefore, the choices regarding the objective funtion are broad.

For the definition of the buckling constraint, it is useful to define a pressure differential $(\Delta p)$, since the riser will be subjected to both internal and external pressures:

$$
\Delta p=p_{i}-p_{e}
$$


As buckling is caused by external pressure (Section 2.2), it only occurs when $\Delta p$ is negative. Since multiple load cases can be considered, the minimum safety factor, corresponding to the maximum pressure differential should be taken, where $m$ is the number of load cases:

$$
S F_{\text {buck }}=\frac{p_{\text {col }}}{\Delta p_{\max }}, \text { where } \Delta p_{\max }=\max \left(-\Delta p_{l}\right), l=1, \ldots, m
$$

In normalized form, the constraint is written as:

$$
1-\frac{S F_{\text {buck }}}{S F_{\text {buck }}^{\text {req }}} \leq 0
$$

where $S F_{b u c k}^{r e q}$ is the minimum Safety Factor required for hoop buckling. This constraint is only included when $\Delta p_{\max }>0$.

To define the laminate failure, the First Ply Failure (FPF) criterion is used [19]. Thus, when the Safety Factor for any lamina reaches the required minimum, the strength constraint is considered active. The Safety Factor of the first ply failure can be defined as:

$$
S F=\min \left(S F_{k l}\right), \text { where } k=1, \ldots, n \text { and } l=1, \ldots, m
$$

In the determination of the Safety Factor for the composite layers, the Tsai-Wu failure criterium is used [19], while for the steel liner the isotropic von Mises failure criterium is adopted. In normalized form, the strength constraint takes the form:

$$
1-\frac{S F}{S F^{r e q}} \leq 0
$$

where $S F^{r e q}$ is the required Safety Factor. In compact form, the optimization model can be given by:

Find $\mathbf{x}=\left[t_{1}, t_{2}, \ldots, t_{n / 2} \quad \theta_{1}, \theta_{2}, \ldots, \theta_{n / 2}\right]$

That minimizes $f(\mathbf{x})=w_{1} \frac{A_{\min }-A}{A_{\min }-A_{\max }}+\left(1-w_{1}\right) \frac{S F_{\text {buck }(\max )}-S F_{\text {buck }}}{S F_{\text {buck }(\max )}-S F_{\text {buck }(\min )}}$

Subjected to:

$$
\begin{aligned}
& 1-\frac{S F_{b u c k}}{S F_{b u c k}^{\text {req }}} \leq 0 \\
& 1-\frac{S F_{\text {comp }}}{S F_{\text {comp }}^{\text {req }}} \leq 0 \\
& 1-\frac{S F_{\text {steel }}}{S F_{\text {steel }}^{\text {req }}} \leq 0
\end{aligned}
$$

Where:

$$
\begin{gathered}
\theta_{k} \in\left\{\theta_{\text {min }}, \theta_{\text {min }}+\Delta \theta, \theta_{\text {min }}+2 \Delta \theta, \ldots, \theta_{\text {max }}\right\} \\
t_{k} \in\left\{t_{\text {min }}, t_{\text {min }}+\Delta t, t_{\text {min }}+2 \Delta t, \ldots, t_{\text {max }}\right\}
\end{gathered}
$$

\section{NUMERICAL EXAMPLES}

This section presents numerical examples in order to study the behavior of the optimization model presented in Section 4 along with the selection and penalty methods showed 
on Sections 3.3 and 3.2. The numerical data used is presented in Table 1. The composite material proprierties are: $E_{1}=137 G P a, E_{2}=9 G P a, \nu_{12}=0.30 G P a, G_{12}=7.1 G P a, F_{1 t}=$ $1517 \mathrm{MPa}, F_{1 c}=1034 \mathrm{MPa}, F_{2 t}=34.48 \mathrm{MPa}, F_{2 c}=151.7 \mathrm{MPa}, F_{6}=0.68 \mathrm{MPa}$. The steel proprierties are: $E=210 G P a, n=0.30, f_{y}=448.2 M P a$. Each problem was solved 30 times. In all examples, two different load cases (A and B) corresponding to extreme situations are considered. Load Case A corresponds to a riser joint on the top of the riser with internal fluid (oil) and the floater at the maximum allowable offset in the Far direction, while Load Case B corresponds to an empty riser joint on the bottom of the sea floor at the maximum allowable offset in the Near direction. Stress constraints are considered in Case A and $\mathrm{B}$ and stability constraints are taken into account only in Case B.

Table 1. Fixed data used in all examples.

\begin{tabular}{|c|c|c|}
\hline \multirow{11}{*}{$\begin{array}{c}\text { Design } \\
\text { Parameters }\end{array}$} & Internal diameter $(m)$ & 0.25 \\
\hline & Number of layers & 10 \\
\hline & Thickness of the inner liner $(m)$ & 0.006 \\
\hline & Internal pressure $(M P a)\left(p_{i}\right)$ & 25.0 \\
\hline & Floater offset & $7.5 \%$ of water depth \\
\hline & Amplification Factor $(\beta)$ & 1.5 \\
\hline & Force Coefficient $\left(\gamma_{f}\right)$ & 1.1 \\
\hline & Thickness of the insulation/protection layer $(\mathrm{m})$ & 0.04 \\
\hline & Specific Weight - internal fluid $\left(k N / m^{3}\right)$ & 7.0 \\
\hline & Specific Weight - sea water $\left(k N / m^{3}\right)$ & 10.05 \\
\hline & Specific Weight - insulation/protection $\left(\mathrm{kN} / \mathrm{m}^{3}\right)$ & 9.0 \\
\hline Design & Allowable ply thickness $(\mathrm{mm})$ & $\{1,2, \ldots, 9,10\}$ \\
\hline Variables & Allowable fiber orientation angles & $\left\{0, \pm 5^{\circ}, \pm 10^{\circ}, 90^{\circ}\right\}$ \\
\hline Required & Buckling & 3.0 \\
\hline Safety & Steel & 1.5 \\
\hline Factors & Composite & 3.0 \\
\hline \multirow{10}{*}{$\begin{array}{c}\text { Genetic } \\
\text { Algorithm } \\
\text { Data }\end{array}$} & Maximum Generations & 1000 \\
\hline & Stallgen & 300 \\
\hline & Individuals per generation & 300 \\
\hline & Analysis and constraint tolerance & $10^{-5}$ \\
\hline & Constant penalty factor $(k)$ & $10^{6}$ \\
\hline & Crossover rate & 0.90 \\
\hline & Mutation rate & 0.01 \\
\hline & Ply addition rate & 0.01 \\
\hline & Ply deletion rate & 0.01 \\
\hline & Swap rate & 0.01 \\
\hline
\end{tabular}

\subsection{Example 1}

In this first example, the three penalty methods (static, Deb's and adaptive) and two selection (Ranking and Fitness Proportional) are used to obtain the optimal solutions. The sea depth and top-angle are fixed at $2500 \mathrm{~m}$ and $20^{\circ}$, respectively. In all optimizations, only three objective function values were obtained. The best objective function reached $(0.1603)$ 
corresponds to a laminate of $22 \mathrm{~mm}$; the second best objective function (0.1776) obtained corresponds to a laminate of $24 \mathrm{~mm}$ and the third objective function (0.1951), which was only found by Deb's Penalty with Ranking Selection, corresponds to a laminate of $26 \mathrm{~mm}$. So, even when the optimum solution was not found, a feasible near-optimum solution was obtained.

Results related to the efficiency of each penalty and fitness method are found on Table 2. The reliability, defined as the number of global optimum solutions found divided by the total of optimizations executed, is showed. The biggest reliability (36.7\%) and smallest average objective function (0.1713) were obtained when using the Static Penalty Method. The use of Deb's Penalty Method with Ranking provided a high reliability (36.7\%), while the combination of Deb's Penalty Method with Fitness Proportional Selection resulted in the worst reliability (13.3\%) with biggest average objective function value. The Adaptive Method showed good results for both types of selection methods. Comparing the Fitness Proportional Selection with Ranking, It is observed that Ranking leads to a faster convergence for all studied cases.

Table 2. Statistical results for 30 optimizations (Ran=Ranking; FP=Fitness Proportional)

\begin{tabular}{cccccccc}
\hline $\begin{array}{c}\text { Penalty } \\
\text { Method }\end{array}$ & $\begin{array}{c}\text { Selection } \\
\text { Method }\end{array}$ & $\begin{array}{c}\text { Reliability } \\
(\%)\end{array}$ & Best & Average & Worst & $\begin{array}{c}\text { Standard } \\
\text { Deviation } \\
(\%)\end{array}$ & $\begin{array}{c}\text { Average } \\
\text { Number of } \\
\text { Generations }\end{array}$ \\
\hline Static & Ran & 36.7 & 0.1603 & 0.1713 & 0.1776 & 8.464 & 349.6 \\
& FP & 36.7 & 0.1603 & 0.1713 & 0.1776 & 8.464 & 363.5 \\
Deb's & Ran & 36.7 & 0.1603 & 0.1719 & 0.1951 & 9.459 & 333.1 \\
& FP & 13.3 & 0.1603 & 0.1753 & 0.1776 & 5.970 & 349.8 \\
Adaptive & Ran & 26.7 & 0.1603 & 0.1730 & 0.1776 & 7.767 & 327.6 \\
& FP & 33.3 & 0.1603 & 0.1719 & 0.1776 & 8.280 & 364.9 \\
\hline
\end{tabular}

Results related to design variables, constraints and objective functions of the optimum solutions are found on Table 3. It is showed that the problem has several optimum solutions and for the majority of them, one active constraint is related to the composite strength Safety Factor since it is near its allowable value in Load Case A. Another almost-active constraint is related to the riser buckling in Load Case B. 
Table 3. Optimum Designs for Example 1 (Sta=Static; Ada=Adaptive; Ran=Ranking; $\mathrm{FP}=$ Fitness Proportional) with $t_{\text {lam }}$ in $\mathrm{mm}$.

\begin{tabular}{|c|c|c|c|c|c|c|c|c|c|}
\hline \multirow{3}{*}{ Pen } & \multirow{3}{*}{ Sel } & & \multirow{3}{*}{$\begin{array}{l}\text { Design } \\
\text { Variables }\end{array}$} & \multicolumn{5}{|c|}{ Safety Factors } & \multirow{3}{*}{$t_{\text {lam }}$} \\
\hline & & & & \multicolumn{2}{|c|}{ Load Case A } & \multicolumn{3}{|c|}{ Load Case B } & \\
\hline & & & & Liner & Comp & Liner & Comp & Buck & \\
\hline \multirow{6}{*}{ Sta } & \multirow{3}{*}{ Ran } & $t$ & {$[4 / 3 / 3 / 1] \mathrm{s}$} & \multirow[t]{2}{*}{1.52} & \multirow[t]{2}{*}{3.04} & \multirow[t]{2}{*}{1.51} & \multirow[t]{2}{*}{12.09} & \multirow[t]{2}{*}{3.00} & \multirow[t]{2}{*}{22} \\
\hline & & $\theta$ & {$[-85 / 60 / 5 /-25] \mathrm{s}$} & & & & & & \\
\hline & & $\begin{array}{l}t \\
\theta\end{array}$ & {$[5 / 1 / 3 / 1 / 1] \mathrm{s}$} & 1.56 & 3.10 & 1.53 & 12.55 & 3.07 & 22 \\
\hline & \multirow{3}{*}{ FP } & $t$ & {$[6 / 1 / 3 / 1] \mathrm{s}$} & & & & & & \\
\hline & & $\theta$ & {$[80 /-50 / 5 /-25] \mathrm{s}$} & 1.53 & 3.02 & 1.54 & 12.57 & 3.06 & 22 \\
\hline & & $\begin{array}{l}t \\
\theta\end{array}$ & $\begin{array}{c}{[6 / 3 / 2] \mathrm{s}} \\
{[85 /-25 / 10] \mathrm{s}}\end{array}$ & 1.53 & 3.02 & 1.55 & 13.13 & 3.14 & 22 \\
\hline \multirow{5}{*}{ Deb } & \multirow{3}{*}{ Ran } & $\begin{array}{l}t \\
\theta\end{array}$ & {$[5 / 1 / 4 / 4] \mathrm{s}$} & \multirow[t]{2}{*}{1.53} & \multirow[t]{2}{*}{3.03} & 1.55 & \multirow[t]{2}{*}{12.76} & \multirow[t]{2}{*}{3.02} & \multirow[t]{2}{*}{22} \\
\hline & & $\begin{array}{l}\theta \\
t\end{array}$ & {$[6 / 1 / 1 / 2 / 1] \mathrm{s}$} & & & & & & \\
\hline & & $\theta$ & {$[80 /-40 / 5 /-5 / 25] \mathrm{s}$} & 1.54 & 3.02 & 1.50 & 11.86 & 3.04 & 22 \\
\hline & \multirow{2}{*}{$\mathrm{FP}$} & $\begin{array}{l}t \\
\theta\end{array}$ & $\begin{array}{c}{[6 / 2 / 1 / 2] \mathrm{s}} \\
{[80 /-35 /-5 / 5] \mathrm{s}}\end{array}$ & 1.54 & 3.06 & 1.54 & 12.59 & 3.04 & 22 \\
\hline & & $\begin{array}{l}t \\
\theta\end{array}$ & $\begin{array}{c}{[3 / 4 / 2 / 1 / 1] \mathrm{s}} \\
{[-85 / 75 /-5 / 0 /-10] \mathrm{s}}\end{array}$ & 1.53 & 3.01 & 1.55 & 12.95 & 3.16 & 22 \\
\hline \multirow{6}{*}{ Ada } & \multirow{3}{*}{ Ran } & $\begin{array}{l}t \\
\theta\end{array}$ & {$[1 / 5 / 4 / 1] \mathrm{s}$} & 1.60 & 3.36 & 1.51 & 12.30 & 3.00 & 22 \\
\hline & & $\begin{array}{l}0 \\
t\end{array}$ & {$[6 / 3 / 2] \mathrm{s}$} & & & & & & \\
\hline & & $\theta$ & {$[80 /-10 /-30] \mathrm{s}$} & 1.52 & 3.06 & 1.54 & 12.67 & 3.01 & 22 \\
\hline & & $t$ & {$[6 / 1 / 2 / 1 / 1] \mathrm{s}$} & 1.56 & 3.09 & 1.51 & 11.94 & 3.01 & 22 \\
\hline & $\mathrm{FP}$ & $\begin{array}{l}\theta \\
t\end{array}$ & {$[3 / 4 / 4] s$} & & & & & & \\
\hline & & $\theta$ & {$[-80 / 75 /-5] \mathrm{s}$} & .53 & 3.03 & 1.56 & 13.29 & 3.08 & 22 \\
\hline
\end{tabular}

\subsection{Example 2}

On the second example, the same data of example 1 is used, but the sea depth is increased to $3000 \mathrm{~m}$. Adaptive Penalty along with Ranking selection were applied. First, the problem was solved using the mono-objective formulation $\left(w_{1}=1.0\right)$, aiming to minimize the composite section area. From the results presented in Table 4, it is noted that the constraint related to the composite material strength is active in Load Case A, while the active constraint in load Case B is the one related to the steel-liner strength. Compared to Example 1, the solutions obtained here present higher values for riser thickness. Differently from the first Example, the constraint related to the riser buckling is not active and every optimum solution found has a different buckling Safety Factor. In order to search the riser with minimum section area and maximum buckling performance, the multiobjective formulation was used.

Comparing the solutions obtained by the mono and multiobjective formulation, the latter produces risers with higher buckling performance, even if both formulations give lam- 
Table 4. Optimum Designs for Example 2 with $t_{\text {lam }}$ in $\mathrm{mm}$.

\begin{tabular}{|c|c|c|c|c|c|c|c|c|}
\hline \multirow{3}{*}{$w_{1}$} & & \multirow{3}{*}{$\begin{array}{l}\text { Design } \\
\text { Variables }\end{array}$} & \multicolumn{5}{|c|}{ Safety Factors } & \multirow{3}{*}{$t_{\text {lam }}$} \\
\hline & & & \multicolumn{2}{|c|}{ Load Case A } & \multicolumn{3}{|c|}{ Load Case B } & \\
\hline & & & Liner & Composite & Liner & Composite & Buckle & \\
\hline \multirow{6}{*}{1.0} & $t$ & {$[1 / 2 / 5 / 8] \mathrm{s}$} & \multirow[t]{2}{*}{1.59} & \multirow[t]{2}{*}{3.00} & \multirow[t]{2}{*}{1.50} & \multirow[t]{2}{*}{13.80} & \multirow[t]{2}{*}{3.35} & \multirow[t]{2}{*}{32} \\
\hline & $\theta$ & {$[35 /-70 /-5 / 85] \mathrm{s}$} & & & & & & \\
\hline & $\begin{array}{l}t \\
\theta\end{array}$ & $\begin{array}{l}{[5 / 5 / 6] \mathrm{s}} \\
{[-70 / 0 / 75] \mathrm{s}}\end{array}$ & 1.58 & 3.07 & 1.50 & 13.19 & 4.89 & 32 \\
\hline & $t$ & {$[7 / 5 / 4] \mathrm{s}$} & \multirow{2}{*}{1.57} & \multirow{2}{*}{3.01} & \multirow{2}{*}{1.50} & \multirow{2}{*}{13.25} & \multirow{2}{*}{6.04} & \multirow{2}{*}{32} \\
\hline & $\theta$ & {$[75 /-5 /-70] \mathrm{s}$} & & & & & & \\
\hline & $\begin{array}{l}t \\
\theta\end{array}$ & $\begin{array}{c}{[7 / 4 / 4 / 1] \mathrm{s}} \\
{[-75 / 70 / 0 / 15] \mathrm{s}}\end{array}$ & 1.57 & 3.00 & 1.50 & 13.21 & 6.83 & 32 \\
\hline \multirow{3}{*}{0.60} & $\begin{array}{l}t \\
\theta\end{array}$ & $\begin{array}{c}{[10 / 2 / 4] \mathrm{s}} \\
{[90 / 15 /-5] \mathrm{s}}\end{array}$ & 1.60 & 3.02 & 1.51 & 13.57 & 7.64 & 32 \\
\hline & $\begin{array}{l}t \\
\theta\end{array}$ & $\begin{array}{c}{[10 / 2 / 1 / 3] \mathrm{s}} \\
{[90 / 15 /-15 /-5] \mathrm{s}}\end{array}$ & 1.59 & 3.00 & 1.51 & 13.61 & 7.65 & 32 \\
\hline & $\begin{array}{l}t \\
\theta\end{array}$ & $\begin{array}{c}{[10 / 1 / 1 / 4] \mathrm{s}} \\
{[90 /-20 /-15 / 5] \mathrm{s}}\end{array}$ & 1.59 & 3.00 & 1.51 & 13.58 & 7.65 & 32 \\
\hline
\end{tabular}

inates with the same total thicknesses $(32 \mathrm{~mm})$. All solutions obtained by the multiobjective formulation shows a slightly increase in the composite strength Safety Factor (1.50 to 1.51) and have its inner and outer layers made of $90^{\circ}$ to increase its collapse external pressure.

\subsection{Example 3}

On the third example, the same data of Example 1 is used, but the top-angle is increased from $20^{\circ}$ to $25^{\circ}$. Adaptive Penalty along with Ranking selection were applied. Results are showed in Table 5. The problem is solved by the mono and multiobjective formulation. Compared to Example 1, the solutions obtained here present higher values for riser thickness. Since the top tension increases with the catenary top angle, the higher axial force makes the composite failure constraint active for Load Case A. The solutions obtained by the multiobjective formulation exhibit a better performance in Load Case B, since all three Safety Factors show an increase in their values. 
Table 5. Optimum Designs for Example 3 with $t_{\text {lam }}$ in $\mathrm{mm}$.

\begin{tabular}{|c|c|c|c|c|c|c|c|c|}
\hline \multirow{3}{*}{$w_{1}$} & & \multirow{3}{*}{$\begin{array}{c}\text { Design } \\
\text { Variables }\end{array}$} & \multicolumn{5}{|c|}{ Safety Factors } & \multirow{3}{*}{$t_{\text {lam }}$} \\
\hline & & & \multicolumn{2}{|c|}{ Load Case A } & \multicolumn{3}{|c|}{ Load Case B } & \\
\hline & & & Liner & Composite & Liner & Composite & Buckle & \\
\hline \multirow{3}{*}{1.0} & $t$ & {$[1 / 5 / 4 / 2] \mathrm{s}$} & 1.52 & 3.03 & 1.51 & 11.79 & 3.00 & 24 \\
\hline & $\theta$ & {$[40 /-80 /-5 / 40] \mathrm{s}$} & & & & & & \\
\hline & $\begin{array}{l}t \\
\theta\end{array}$ & $\begin{array}{c}{[5 / 2 / 2 / 3] \mathrm{s}} \\
{[75 / 10 /-5 /-45] \mathrm{s}}\end{array}$ & 1.50 & 3.01 & 1.51 & 11.50 & 3.22 & 24 \\
\hline \multirow{2}{*}{0.60} & $\begin{array}{l}t \\
\theta\end{array}$ & $\begin{array}{c}{[6 / 2 / 4] \mathrm{s}} \\
{[90 /-15 / 10] \mathrm{s}}\end{array}$ & 1.53 & 3.02 & 1.54 & 12.68 & 3.91 & 24 \\
\hline & $\begin{array}{l}t \\
\theta\end{array}$ & $\begin{array}{c}{[6 / 2 / 1 / 3] \mathrm{s}} \\
{[90 /-15 / 15 / 10] \mathrm{s}}\end{array}$ & 1.52 & 3.00 & 1.54 & 12.70 & 3.91 & 24 \\
\hline
\end{tabular}

\section{CONCLUSIONS}

This paper presented a methodology for optimization of composite catenary risers, using a Genetic Algorithm developed specially crafted for laminated structures. Different penalty methods and fitness functions were employed. For the studied cases, the penalty methods without control parameters were able to find optimum solutions. The use of Ranking led to a faster convergence than the Fitness Proportional Selection method. It was observed that for all cases, the constraint related to the composite strength Safety Factor was active in Load Case A. In Load Case B the composite strength Safety exhibited a high Safety Factor.

Several different composite risers with the same thickness different buckling pressures were obtained. So, a multiobjective formulation was used successful to achieve a riser with minimum thickess and maximum buckling pressuree. As expected, the solutions obtained by the multiobjective formulation exhibited a better perfomance to buckling.

\section{Acknowledgements}

The financial support by CNPq (Conselho Nacional de Desenvolvimento Científico e Tecnológico) and CAPES (Coordenação de Aperfeiçoamento de Pessoal de Nível Superior) are gratefully acknowledged.

\section{References}

[1] M. M. Salama. Some challenges and innovations for deepwater developments. In: Offshore Technology Conference, OTC 8455, 1997.

[2] P. J. C. Tamarelle and C. P. Sparks. High-performance composite tubes for offshore applications. In: Offshore Technology Conference, OTC 5384, 1987.

[3] K. L. Smith and M. E. Leveque. Ultra-deepwater production systems final report. Technical report, 2005. 
[4] O. O. Ochoa and M. M. Salama. Offshore composites: Transition barriers to an enabling technology. Composites Science and Technology, 2005.

[5] L. C. M. Meniconi, S. R. Reid, and P. D. Soden. Preliminary design of composite riser stress joints. Composites Part A: Applied Sciences and Manufacture, 32:597-605, 2001.

[6] F. A. F. Teófilo. Análise e projeto de risers compósitos em catenária. Master's thesis, Federal University of Ceará, Brazil, 2010.

[7] Z. Gurdal, R. T. Haftka, and P. Hajela. Design and Optimization of Laminated Composite Materials. John Wiley \& Sons, 1999.

[8] F. S. Almeida and A. M. Awruch. Design optimization of composite laminated structures using genetic algorithms and finite element analysis. Composite Structures, 88:443-454, 2009.

[9] E. Potgieter and N. Stander. The genetic algorithm applied to stiffness maximization of laminated plates: review and comparison. Structural Optimization, 15:221-229, 1998.

[10] J. H. Park, J. H. Hwang, C. S. Lee, and W. Hwang. Stacking sequence design of composite laminates for maximumstrength using genetic algorithms. Composite Structures, $52: 217-231,2001$.

[11] R. T. Marler and J. S. Arora. Survey of multi-objective optimization methods for engineering. Structural and Multidisciplinary Optimization, 26:369-395, 2004.

[12] M. Walker and R. E. Smith. A technique for the multiobjective optimisation of laminated composite structures using genetic algorithms and finite element analysis. Composite Structures, 62:123-128, 2003.

[13] C. M. Larsen and T. Hanson. Optimization of catenary risers. Journal of Offshore Mechanics and Arctic Engineering, 121:90-94, 1999.

[14] R. L. Tanaka and C. A. Martins. Parallel dynamic optimization of steel risers. Journal of Offshore Mechanics and Arctic Engineering, 133, 2011.

[15] I. N. Vieira, B. S. L. P. Lima, and B. P. Jacob. Optimization of steel catenary risers for offshore oil production using artificial immune system. Lecture Notes in Computer Science, 5132:254-265, 2008.

[16] A. A. Pina, C. H. Albrecht, B. S. L. P. Lima, and B. P. Jacob. Tailoring the particle swarm optimization algorithm for the design of offshore oil production risers. Optimization and Engineering, 12(1-2):215-235, 2010.

[17] B. S. L. P. Lima, B. P. Jacob, and N. F. F. Ebecken. A hybrid fuzzy/genetic algorithm for the design of offshore oil production risers. International Journal for Numerical Methods in Engineering, 64:1459-1482, 2005.

[18] H. Yang, R. Jiang, and H. Li. Optimization design of deepwater steel catenary risers using genetic algorithm. Computational Structural Engineering, pages 901-908, 2009.

[19] I. M. Daniel and O. Ishai. Engineering Mechanics of Composite Materials. Oxford University Press, 2nd edition, 2006. 
[20] F. A. F. Teófilo, E. Parente Jr, A. M. C. Melo, and A. S. Holanda. Análise de placas laminadas pelo método dos elementos finitos. In: XXIX Iberian Latin-American Congress on Computational Methods in Engineering, pages 1-21, 2008.

[21] R. Cook, D. Malkus, M. Plesha, and R. J. de Witt. Concepts and Applications of Finite Element Analysis. John Wiley \& Sons, 2002.

[22] J. N. Reddy. Mechanics of Laminated Composite Plates and Shells: Theory and Analysis. CRC Press, 2nd edition, 2004.

[23] C. P. Sparks. Fundamentals of Marine Riser Mechanics: Basic Principles and Simplified Analysis. PennWell Books, 2007.

[24] DNV - Det Norske Veritas. DNV-OS-F201 - Dynamic Risers - Offshore Standard, 2001.

[25] Buckling of Thin-Walled Circular Cylinders. NASA SP-8007, Space Vehicle Design Criteria (Structures), 1968.

[26] DNV - Det Norske Veritas. DNV-RP-F202 - Composite Risers - Recommended Practice, 2003.

[27] H. J. C. Barbosa and A. C. C. Lemonge. An adaptive penalty scheme for genetic algorithms in structural optimization. International Journal for Numerical Methods in Engineering, 59:703-736, 2004.

[28] K. Deb. An efficient constraint handling method for genetic algorithms. Computer Methods in Applied Mechanics and Engineering, 186:311-338, 2000.

[29] D. E. Goldberg. Genetic Algorithms in search, Optimization and Machine Learning. Addison-Wesley, 1989.

[30] F. A. Sadjadi. Comparison of fitness scaling functions in genetic algorithms with applications to optical processing. In: Proceedings of SPIE, 5557, 2004.

[31] R. Kicinger, T. Arciszewski, and K. De Jong. Evolutionary computation and structural design: A survey of the state-of-art. Computers and Structures, 83:1943-1978, 2005.

[32] O. Erdal and F. O. Sonmez. Optimum design of composite laminates for maximum buckling load capacity using simulated annealing. Composite Structures, 71:45-52, 2005.

[33] A. R. M. Rao and P. P. Shyju. A meta-heuristic algorithm for multi-objective optimization design of hybrid laminate composite structures. Computer-Aided Civil and Infrastructure Engineering, 12(1-2):149-170, 2010.

[34] E. J. Barbero. Introduction to Composite Material Design. CRC Press, 1999.

[35] A. W. Blom, P. B. Stickler, and Z. Gurdal. Optimization of composite cylinder under bending by tailoring properties in circunferential direction. Composites: Part B, 41:157$165,2010$.

[36] J. Arora. Introduction to Optimum Design, volume 2nd. Elsevier, 2nd edition, 2004.

[37] S. Adali, V. E. Verijenko, and A. Richter. Minimum sensitivity design of laminated shells under axial load and external pressure. Composite Structures, 54:139-142, 2001. 